\title{
A Cognitive Linguistic Approach to Generics
}

\author{
Zhengling $\mathrm{Fu}^{1}$ \\ ${ }^{1}$ College of International Studies, Southwest University, Chongqing, China \\ Correspondence: Zhengling Fu, College of International Studies, Southwest University, Chongqing, China. \\ Email: rainbow9119@163.com
}

$\begin{aligned} & \text { Received: January 26, } 2015 \quad \text { Accepted: February 16, } 2015 \quad \text { Online Published: March 29, } 2015 \\ & \text { doi:10.5539/ijel.v5n2p115 }\end{aligned}$ URL: http://dx.doi.org/10.5539/ijel.v5n2p115

\begin{abstract}
As a special sentence type, generic sentences contribute a lot to the understanding of natural languages and have been a concern of semanticists, pragmatists and philosophers. In view of inefficiency of existing theoretical framework on generics, this paper addresses generics in the framework of cognitive linguistics. A type is a fictive entity, not an actual individual. It represents an abstraction from actuality which captures the commonality inherent across a set of actual instances. Conceptualization of generics is dynamic in nature. Generics' syntax features and exceptions are both the natural result of cognitive processing, and truth conditions should be judged in terms of certain ICMs rather than the correspondence between linguistic expressions and reality of the physical world. It is concluded that fictivity, dynamicity and ICM (Idealized Cognitive Model) are three cognitive motivations, which are the answers to the puzzles of generics' tolerating exceptions.
\end{abstract}

Keywords: cognitive linguistics, generics, dynamicity, fictivity, ICM

\section{Introduction}

Generic sentences, in all languages, express generalization (Greenberg, 2003) referring to such sentences as Birds fly, Chickens lay eggs and Ravens are black. Generics describe the general property of instances in a category, not property of a specific individual.

The research of generics can be traced back to 1970s, and in view of its significance in thought and language, researchers from various fields study it from various perspectives such as logics, artificial intelligence, philosophy and psychology, though up to now no conformably accepted theory has been set up. The recent two or three decades witnessed the deep study on generic sentences which is "a comparatively new research field" in linguistics (Chung, 2002, p. 203). Previous studies (Carlson \& Pelletier, 1995; Cohen, 1999; Eckardt, 1999; Greenberg, 2003) focus on the definition, types, property, logical analysis, truth value analysis, definite nouns and indefinite nouns of subject, the application of singular and plural nouns, the application of adverbs of frequency and their revelation on the study of non - monotonic logical reasoning and artificial intelligence.

Cognitive linguistics... is an approach to language that is based on our experience of the world and the way we perceive and conceptualize it (Ungerer \& Schmid, 1996, p. x). Within the framework of cognitive linguistics, generics will obtain a comprehensive study from a new perspective. This paper makes efforts to reveal cognitive motivation of generics, holding that generics' economy expression and incomplete statements are closely related to human's innately given cognitive capacity, and communicating economically with least efforts and least expression is universally accepted rather than the vice versa. It is claimed that generics' syntax features and exceptions are both the natural result of cognitive mechanisms, and truth conditions should be judged in terms of certain ICMs rather than the correspondence between linguistic expressions and reality of the physical world.

\section{Generics: A Characterization}

\subsection{Features of Generics}

Three critical features are found in generic sentences: 1) generic sentences expressing regularity have universality. Generics express the general property of a kind, such as Dogs bark, not This dog barks. Property in generic sentences can hold of instances in a certain domain. For example, Dogs bark can lead to the conclusion "this dog barks". Hence there is some similarity between generic sentences and universal sentences, and generics can be regarded as universal sentences to some degree. 2) Generics can tolerate exceptions. Compared with universal sentences, the main feature of generics is its exception-tolerating property. For example, the sentence 
Chickens lay eggs appears to be a truism, yet most people are well aware that cocks do not lay eggs, and theoretically this should falsify the statement-yet it does not seem to. This is an example of "exception tolerance". 3) Generics are connotational. Even no instance in the real world can satisfy the predicate, generics can still be true. For example, there is no "dragon" in the real world, the sentence Dragon is a symbol of power can still be true in light of China's culture. Due to the above features, the study of generic sentences is not only the key research subject in philosophy, science of cognition, logic (epistemic logic and non-monotonic logic) and artificial intelligence, but a breakthrough to categorization of semantics and reasoning of pragmatics.

\subsection{Classification of Generics}

According to Greenberg (2003), the class of generic sentences is not only syntactically but also semantically heterogeneous. Krifka (1987) once introduced one such distinction, originally labeled the D (definite)/ I (indefinite) genericity distinction, also called kind-referring sentences and characterizing sentence respectively. Krifka's (1987) distinction between I-generics and D-generics is particularly useful. D-generics predicate properties of their subjects that do nothold of particular members of a kind, but only of a kind itself. I-generics predicate properties of their subjects that can hold of particular members of the kind. Examples are as follows:

D-generics:

The Dodo is extinct.

Dogs are widespread.

Dinosaurs died out many years ago.

I-generics:

Dogs bark.

A cat drinks milk.

The tiger is striped.

D-generic sentences assert that a certain property holds directly of the kind itself. For example, sentence Rats were reaching Australia in 1770 seems to mean that the kind "rat" reached Australia in 1770, and sentence Dodos are extinct means that the whole kind "dodo" is extinct. Kind-referring generics have kind-referring NPs, which does not denote or designate any particular persons or things, but rather the kind of people or things.

The other phenomenon related to genericity is propositions which do not denote specific or separated events, but rather some general property, that is, regularity based on many specific events and facts. In such case, genericity is the property of the whole sentence, not the property of any NPs of the whole sentence. The whole sentence expresses regularity, which transcends particular events or facts. This paper is concerned with only this kind of genericity.

\subsection{Two approaches to Generics}

Generic sentences have intrigued scholars since at least the 17th century. It is not surprising, then, that there are a vast number of approaches to this problem, from widely different points of view. A useful classification of the various approaches into two broadly defined camps is made by Carlson (1995). Carlson distinguishes between "the rules and regulations approach" and "the inductivist approach" (p. 225).

"Rules and regulations" approach relies on, for example, laws of nature and genetics to ascertain the truth of a statement like Crows are black. That is to say, the truth of generics is not the result of instances' properties, but evaluated by rules and regulations which are "basic", "irreducible". Hence, a sentence is either true or false. These so-called rules and regulations may be physical, biological, social, moral, and etc. Although Calson (1995) regarded this approach true, he, as well, pointed that this approach faces several problems: in terms of ontology, "rules and regulations" approach is redundant-it requires the existence of entities, namely rules and regulations, which do not seem to be needed on independent grounds.

According to the inductivist approach, a generic sentence is true if "sufficiently many" "relevant" individuals in the domain of the generic satisfy the predicated property. There are many specific types of inductivist theory, which vary mainly depending on which instances count as "relevant" (e.g., contextually restricted individuals, "normal" individuals, "typical" individuals, etc.) and how many is considered "sufficiently many" (e.g., all, most, "substantially many" etc.). Anyone who proposes that genericity involves quantification is committed to some version of the inductivist view. "A problematic class of sentences for the inductivist approach to generics is precisely the kind which the rules and regulations handle with ease, namely explicit conventions". For example, Bishops move diagonally is undoubtedly true because we are told this rule before we play chess, rather than arriving at such a conclusion after much watching such game. 
The inductivist approach...appears to insist on some very unrealistic scenarios regarding how we come to judge a generic as true or false. For example, the fact is that we learn most rules of games we play not by watching some else play...but rather by being told what the rules are. When I learned the rules of chess, I did not observe a bishop moves diagonally. And none of us, to be sure, learned that bachelors are unmarried by observing bachelors and then noting the strong tendency for them not to be married. Instead, we found these things out in other ways (Calson, 1995, pp. 230-231).

Perhaps the most severe criticism of the inductivist approach is the grave difficulty of finding an acceptable general definition of how many instances are sufficiently many.

\section{Cognitive Motivations in Generics}

A natural language is any language which arises, unpremeditated, in the brains of human beings. Human beings use natural languages to communicate with each other. They are distinguished from constructed and formal languages such as those used to program computers or to study logic. Traditional approaches to semantics consist of assigning things like objects, properties, senses, or truth conditions to sentences and their parts. These more traditional approaches to semantics might be viewed as dealing with the question of how language is related to the world. This is an interesting question, and has received much attention. There are, however, different questions related to meaning that we might wish to address: how do human beings actually understand their natural languages? What are the mental processes that underlie our ability to comprehend and produce the sentences of our languages?

\subsection{Fictivity}

Language has long been used as a tool to describe the world around us. Canonically, therefore, nominal expressions would be used for the direct description of actual individuals, and sentences for the direct description of actual events and situations they participate in. Yet Langacker (2003) thought this view may not be accurate. "Departures from the supposed canon are so varied and prevalent as to suggest a fundamental revision in how we think about language and the functions it serves. Such cases are not limited to the kinds of non-actuality involved in making false statements, in describing future events (which might not actually eventuate), or in creating fictitious worlds (as in a novel). Indeed, one has to be struck by how very common it is that fictitious entities are invoked and directly described even when our concern is with actuality. Surprisingly often, our characterization of actual situations is effected only indirectly, through the mediation of fictive or virtual entities conjured up for that purpose." (Langacker, 2003, pp. 1-64)

Note first that, by itself, a lexical noun (e.g., cat, oxygen) merely specifies a type of thing, not any particular instance of that type. The entity (thing or process) designated by a type specification is fictive in nature; it per se does not refer to an actual individual. It is only at the level of a full noun phrase (e.g., this cat, some oxygen) that reference is made to particular instances of a thing.

A type is a fictive entity, not an actual individual. It represents an abstraction from actuality which captures the commonality inherent across a set of actual instances. A thing type corresponds to any number of instances of that type, distinguished by their position in the domain of instantiation. It is important to keep in mind how types (and other kinds of virtual entities) are connected to actuality, as well as how they arise from it. Types arise as a kind of generalization over actual occurrences, such that sets of occurrences are perceived as being alike in significant respects.

To briefly illustrate some of these points, let us consider the following expression (with the following figure):

(1) A bird flies.

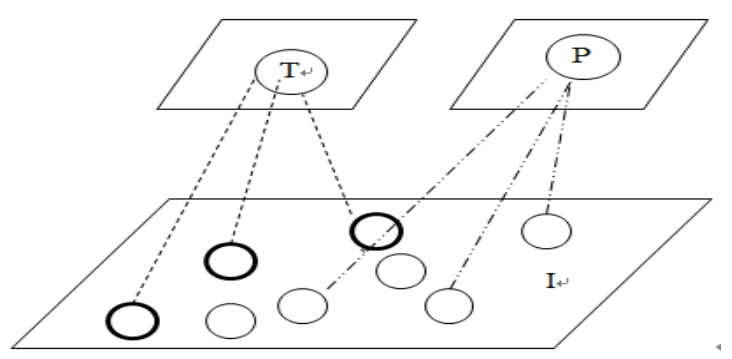

Figure 1. Fictive representation of sentence (1) 
The subject "a bird" is a fictive (or virtual) entity, not an actual instance of the type, which can be represented by $\mathrm{T}$ or $\mathrm{P}$ in the above figure. The rectangle "I" in the above figure, as an abstract container, refers to the instances set of "birds". "T" stands for the fictive concept "birds", and "P" also stands for fictive concept "birds". The dashed lines connected to "T" and "P" are different, which means that although "T" and "P" both belong to the type plane, they are abstracted from instances by different ICM, different situations, different salient features, and so on. Thus, the subject "a bird" is an imagined instance, one "conjured up" in order to make a generalization that covers relevant actual instances. " $T$ " plane stands for the generalization of birds' flying ability, while "P" plane may stand for generalization of other properties, such as "has wings" "has feathers", and so on. That is to say, for plane "T", the fictive concept "birds" is abstracted from some instances in the plane "I" which can best represent birds' ability of flying. The relevant instances here are profiled by heavy lines, while other instances are background with light lines. In such case, other features as "has wings", "has feathers" or those instance which cannot fly, are backgrounded, while instances satisfying the features are foregrounded. The foreground part, according to our encyclopedic knowledge, constitutes a prominent part of the global knowledge concerning "birds", and then this generic sentence get legitimacy, becoming a global generalization pertaining to what is conceived as the world's stable structure. And the other instances, the backgrounded ones, may be the source of another fictive plane "P". So the concepts in plane "T" or plane "P" are both fictive, and they do not correspond to any physical instances, but an abstraction, a generalization from instances. In the whole process of conceptualization, no particular bird is involved, but those virtual ones inhabiting a special mental space invoked make a generalization concerning birds. The genericity is not specifically coded, but inheres in the nature of the conceived relationship between actuality and the abstract container in which the profiled event is found.

Langacker stated that it is a virtual instance of the type conjured up for purposes of making a generalization. Reference to a single instance is sufficient for this purpose provided that this instance is somehow taken as being representative of the class as a whole. It is through such representativeness that a property ascribed to a single (fictive) instance is inferred as being universally applicable to class members.

There is another kind of fictivity in genericity, exemplified in the following sentence:

(2) Firemen fight fires.

Even if there are no fire at a certain time point, but we all hold an expectation that firemen are supposed to fight fires at any time. It is true that "fighting fires" is not an entity in reality, but it represents an expectation since it is the job of firemen. It means that in case of fires, firemen should and are ready to fight fires. This fictivity is a clear example of embodiment, reflecting various aspects of our everyday experience in dealing with the physical world, though no actuality is mentioned.

\subsection{Dynamicity}

Because it resides in neurological activity, conceptualization is necessarily dynamic (Langacker, 1999). In this section, dynamicity means that it emerges and develops through processing time and sentence with similar word order may have different path of conceptualization and produce different truth value and different interpretation.

According to Langacker, the most obvious cases of dynamicity are those correlated with word order. Due to the temporality of the speech stream, we can hardly avoid accessing facets of a complex conception in the order given by the sequencing of the words that symbolize them. Dynamicity is not, however, limited to this dimension. It is essential that we do not oversimplify the manifest complexity of language processing by a single "left-to-right" linear order. Instead, we can reasonably presume that sequenced processing occurs simultaneously in multiple dimensions and on different time scales.

Let's focus on the following two sentences once again:

(3) Chickens lay eggs.

(4) Chickens are female.

Mental process of sentence (3) can be depicted in figure 2 . 


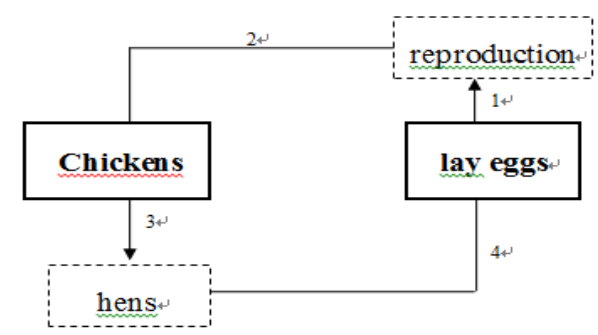

Figure 2. Construal process of sentence (3)

In the above figure, the generic sentence Chickens lay eggs is put in solid square frame. The predicate "laying eggs" in step 1 activates the domain "reproduction" in dotted square frame, which determines the construal direction in step 2. With this restriction, in step 3 chickens is metonymically understood as hens in dotted square frame, since it is hens that lay eggs. In step 4, "hens lay eggs" is definitely true. The whole construal process is continuous and reasonable.

The construal process of sentence (4) is depicted in Figure 3. The subject "chickens" plays the same function as sentence (3), however, the predicate "are female" in step 1 determines the focus of processing as "gender". As to gender, chickens are composed of cocks and hens. While in step 4, the connection between "cocks and hens" and "are female" is blocked since cocks are definitely male.

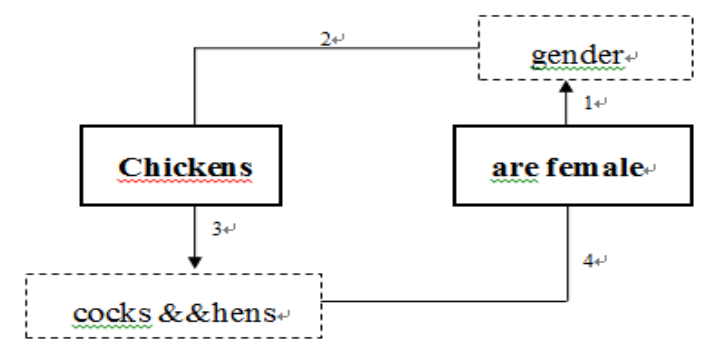

Figure 3. Gender relationship of sentence (4)

\subsection{Generics and ICMs}

\subsubsection{Characteristic Dimensions}

It is clear that generics ought to be an efficient information gathering mechanism, since it is our most basic and immediate means of obtaining information about categories. One way genericity might be efficient is for it to take advantage of regularities out there in the world. Characteristic dimensions of animal kinds include perhaps noises, modes of locomotion, means of reproduction and gestation, and diet; characteristic dimensions of artifact and social kinds may include function and role respectively.

For example, we expect that biological kinds will exhibit certain characteristics, or else face extinction. The most obvious expectation of biological kinds is that reproduction will be possible. It is common knowledge that for an animal kind to survive, certain conditions must be met.

The following CMs should be taken into consideration:

(I). There must be both male and female members of the species.

(II). There must be a manner in which this reproduction and subsequent gestation occurs.

(III). There must be adult members of the species.

(IV). The young must be nourished in some way.

We can rewrite ICMs for REPRODUCTION into:

$$
\text { REPRODUCTION-ICM }=C M(I)+C M(I I)+C M(I I I)+C M(I V)
$$

Once generalization has identified a characteristic dimension for a domain of kinds, it will then seek to fill in a value along the dimension for a given kind within the domain. Having identified reproduction as a characteristic dimension of animal kinds, we can fill in the value for ducks upon learning of the egg-laying ducks. The existence of even just a few egg-laying ducks will suffice to provide a value for this dimension. Since all we are 
looking to do is locate a value along a dimension, no statistical information about the percentage of ducks that lay eggs, etc, is needed. The fact that less than half of ducks lay eggs has no impact on this generalization. While a neglect of one CM may lead to falsity of the sentence, for example, Chickens are female is false, since CM(I) is neglected. The same holds for sentence like Books are paperback. Since this sentence needs an ICM related to books material and appearance, those books with hardcover should not be neglected. The sentence Books are paperback needs setting up the following ICMs:

(I) Books take some form of binding.

(II) Books include paperbacks and hardcovers.

(III) The number of paperbacks is much larger than hardcovers.

In such ICMs, it is true that the number of paperbacks is much larger than that of hardcovers, we still cannot say Books are paperbacks since there exists an obvious alternative to paperback, that is "hardcover", no matter what the ratio is.

An efficient information gathering system could use information from a related category to predict characteristic dimensions of a novel one, and then use even a single instance of the novel category to obtain the value of that characteristic dimension for the new category.

\subsubsection{Striking Features}

A large number of generic sentences do not pertain to characteristic dimensions, but predicate striking properties. Under that circumstance, sentences attribute harmful, dangerous, or appalling properties to kind. Take the sentence Mosquitoes carry $W N V$ for example. The following CMs are essential to judge this sentence.

(I) There exists West Nile Virus.

(II) Both human being and animals can carry WNY (West Nile Virus).

(III) Mosquitoes are disposed to carry WNY (West Nile Virus).

(IV) A small number of mosquitoes carrying WNY (West Nile Virus).

Based on the above CMs, the statement Mosquitoes carry WNY is intuitively true, even if the number of those carrying the virus is very small, in that this sentence aims to describe the animal kind "mosquitoes" has such disposition, rather than to state the statistical advantage of mosquitoes' carrying the virus.

Another typical example is $A$ turtle lives a long life. In real world, most turtles die in infancy, only a few may survive to a long life. The truth of this sentence can still be supported by the following ICMs:

(I) Life span varies from one animal kind to another.

(II) Most turtles die in infancy.

(III) In satisfying conditions, some turtles live much longer lives than other animals.

The ICMs is to reveal turtle's speciality in life span that - turtles have the characteristic of living long lives, so though most turtles die in infancy, the fact that some turtles can live long lives can suffice to verify the truth of $A$ turtle lives a long life. If the sentence is changed into Turtles all live long lives, then corresponding ICMs should be modified into:

(I) Life span varies from one animal kind to another.

(II) No turtles die in infancy.

(III) It is true for all turtles to live long lives.

In such ICMs, one exception to long-living turtles will make the sentence All turtles live long lives false, in that $\mathrm{CM}(\mathrm{II})$ makes a strict restriction on it.

ICMs are open-ended and tolerant to exceptions, as Lakoff (1987, p. 132) stated "ICMs are cognitive models that are idealized. They do not have to fit the world and they can be used by the speakers to suggest how to, or not to, understand a given situation."

It is evidently found that the more striking, appalling, or otherwise gripping we find the property predicated in the generic, the more tolerant the generic is to exceptions. It is sufficient for the truth value of the generic that just some of the members of the kind have the property in question.

The truth judgment of generics cannot proceed without ICM, in that generics might become troublesome with the influence of exceptions. Only with the restriction of ICM, generic sentences can get their truth value with 
neglecting possible exceptions in the real world. Aiming to generalize knowledge of the world around us, generic sentences per se should be judged with different ICM since their references are different facets of the world. ICMs like stereotypes, paragons and ideals are metonymic in the sense that a single type or individual stands for the entire category.

\section{Conclusions}

What is invoked by language is fictive in nature, since it stems from people's embodiment in the world, instead of corresponding to the physical world. Generics is such a typical language form whose truth conditions do not depend on the correspondence to the physical world. Therefore, some meanings are not conveyed directly by language, but activated by language. What needs to be mentioned is that the activation of similar sentence pattern is not necessarily the same, but dynamic in processing time and processing dimensions. Much default meaning is conveyed through these cognitive processing. According to Chomsky (2000), similar phenomena exist elsewhere in language. The sentence John climbed the mountain is by default understood as "John climbed up the mountain" instead of "John climbed down the mountain". To obtain the latter interpretation, an explicit use of "down" should not be omitted. The unmarked use of "climbed the mountain" is never interpreted as climbed down. That is why we usually say Chickens lay eggs rather than Hens lay eggs, or Cocks never lay eggs. If there is a case of gene mutation that a cock lays eggs, then we might describe it as A chicken lays eggs and it is a cock.

This study is an attempt to systematically explore motivations and legitimacy of generic sentence under the framework of cognitive linguistics. However, generics cover a very wide range, this paper only focuses on characterizing generics, and kind-referring generics are beyond the discussion of this dissertation. In order to get a comprehensive understanding of generics, other types of generics should also be studies in great details with the theoretical support of cognitive linguistics in future research.

\section{Acknowledgements}

This paper is supported by the following projects: The Fundamental Research Funds for the Central Universities (SWU1509176), The Education and Teaching Reform Project (2013JY094).

\section{References}

Carlson, G., \& Pelletier, F. (1995). The Generic Book. Chicago: The University of Chicago Press.

Chomsky, N. (2000). New Horizons in the Study of Language and Mind. Cambridge: Cambridge University Press. http://dx.doi.org/10.1017/CBO9780511811937

Chung, K. S. (2002). Book review of Think Generic: The meaning and use of generic sentences. Language, $78(1), 203$.

Cohen, A. (1999). Think Generic! The Meaning and Use of Generic Sentences. Chicago: The University of Chicago Press.

Eckardt, R. (1999). Normal objects, normal worlds and the meaning of generic sentences. Journal of Semantics, 3, 237-278. http://dx.doi.org/10.1093/jos/16.3.237

Greenberg, Y. (2003). Manifestations of Genericity. London: Routledge.

Krifka, M. (1987). An Outline of Genericity [partly in collaboration with C. Gerstner], SNS-Bericht, University of Tubingen.

Lakoff, G. (1987). Women, Fire, and Dangerous Things: What Categories Reveal about the Mind. Chicago: The University of Chicago Press. http://dx.doi.org/10.7208/chicago/9780226471013.001.0001

Langacker, R. (1999). Foundations of Cognitive Grammar: Theoretical Prerequisites. California: Stanford University Press.

Langacker, R. (2003). Dynamicity, Fictivity, and Scanning: The Imaginative Basis of Logic and Linguistic Meaning. Korean Linguistics, 18, 1-64.

Radden, G., \& Kovecses, Z. (1999). Towards a theory of metonymy. In K-U. Panther \& G. Radden (Eds.), Metonymy in Language and Thought. Amsterdam/ Philadelphia: John Benjamin's Publishing Company. http://dx.doi.org/10.1075/hcp.4.03rad

Ungerer, F., \& Schmid, H. J. (1996). An Introduction to Cognitive Linguistics. London: Longman. 


\section{Copyrights}

Copyright for this article is retained by the author(s), with first publication rights granted to the journal.

This is an open-access article distributed under the terms and conditions of the Creative Commons Attribution license (http://creativecommons.org/licenses/by/3.0/). 